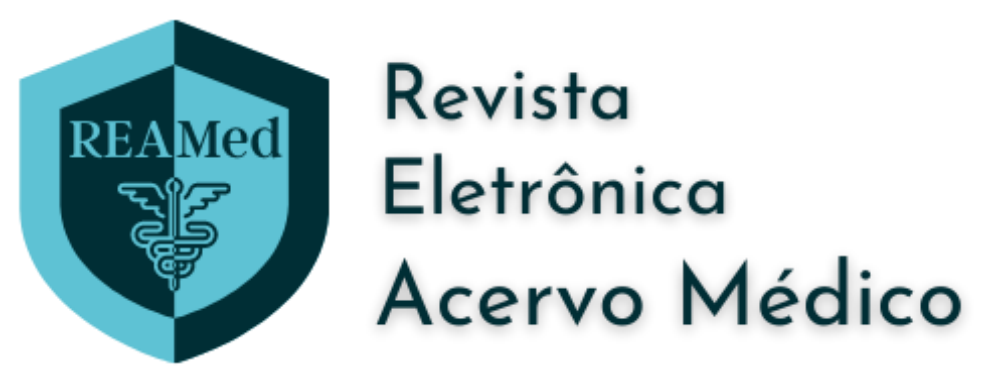

\title{
Abordagem geral da doença renal crônica e sua relação com a hipertensão arterial sistêmica: uma revisão integrativa
}

\author{
General approach towards chronic kidney disease and it's relation with systemic arterial \\ hypertension: an integrative review \\ Enfoque general de la enfermedad renal crónica y su relación con la hipertensión arterial \\ sistémica: una revisión integradora
}

João Wilton Lucena Bessa1, Francisco Davi Fernandes Brilhante ${ }^{2}$, Gabriela de Oliveira Borges ${ }^{3}$, Jonatan Lucena Bessa ${ }^{1}$, Maria Eduarda Geddo Kostakis ${ }^{4}$, Pábulo Diego de Oliveira de Sousa ${ }^{5}$, Priscila Araújo e Silva ${ }^{1}$, Thamirys Sartori de Souza ${ }^{6}$, Victor Talles de Melo Fontenelle ${ }^{7}$, Raquel de Oliveira Monteiro Tavares ${ }^{8}$.

\section{RESUMO}

Objetivo: Enriquecer a literatura, sintetizar e esclarecer a relação da Doença Renal Crônica (DRC) com a hipertensão. Métodos: Essa revisão integrativa utilizou-se as bases de dados "PubMed" e "Scielo" para procura dos descritores "Renal Function", "Hypertension", "Kidney Function", "Renal Insufficiency, Chronic" e "Chronic Kidney Disease" associado com operadores booleanos, encontrando 1116 artigos. Destes, selecionou-se 20. Resultados: A DRC apresenta alta mortalidade devida seu caráter silencioso ou inespecífico. É caracterizada por anormalidades funcionais e/ou estruturas dos rins por mais de três meses. A Taxa de Filtração Glomerular é utilizada para diagnóstico e estadiamento e, como principais etiologias, tem-se a hipertensão e diabetes, sendo doenças foco para tratamento da DRC. Porém, não há explicação simples e fidedigna para essa relação, e ainda há estudos relatando que o tratamento intensivo da pressão e o uso de hidralazina podem piorar a função renal. Todavia, os anti-hipertensivos, medicamentos de controle glicêmico e modificadores de lipídios são clássicos como terapia modificadora da progressão. Considerações finais: É imprescindível mais ênfase no rastreamento e diagnóstico precoce para melhorar a saúde dos pacientes, principalmente aqueles com comorbidades. Ainda, mais estudos são necessários para elucidar sua relação com a hipertensão, o tratamento intensivo da pressão e outras associações.

Palavras-chave: Doença renal crônica, Hipertensão, Pressão arterial.

\section{ABSTRACT}

Objective: Complement the literature, summarize the subject and clarify the relation between Chronic Kidney Disease (CKD) and hypertension. Methods: This integrative review used "PubMed" and "Scielo" databases to search for the "Renal Function", "Hypertension", "Kidney Function", "Renal Insufficiency, Chronic" and "Chronic Kidney Disease" descriptors associated with Boolean operators, resulting in 1116 articles, in which 20 of them were chosen. Results: CKD has a high

\footnotetext{
${ }^{1}$ Universidade de Fortaleza (UNIFOR), Fortaleza - CE. ${ }^{\star}$ E-mail: joaowilton@edu.unifor.br

2 Centro Universitário Christus (UNICHRISTUS), Fortaleza - CE.

${ }^{3}$ Universidade Nove de Julho (UNINOVE), São Paulo - SP.

${ }^{4}$ Universidade São Caetano do Sul (USCS), São Paulo - SP.

5 Faculdade Atenas (UNIATENAS), Sete Lagoas - MG.

${ }^{6}$ Centro Universitário São Camilo, São Paulo - SP.

${ }^{7}$ Universidade de Potiguar (UnP), Natal - RN.

8 Universidade Federal Fluminense, Niterói - RJ.
} 
mortality rate due to its silent or nonspecific character. It is characterized by functional and/or structural kidney abnormalities for more than three months. The glomerular filtration rate is used for diagnosis and staging and, as main etiologies, hypertension and diabetes are the most common ones. However, there is no simple and reliable explanation for the direct physiopathogenesis. Antihypertensive drugs, glycemic control drugs and lipid modifiers are essential for progression-modifying therapy, although there are studies reporting that intensive pressure treatment and the use of hydralazine can worsen renal function. Final considerations: More emphasis on screening and early diagnosis is essential to improve the health of patients, especially those with comorbidities. Therefore, more studies are needed to elucidate its relation with hypertension, intensive pressure treatment and other associations.

Key words: Chronic kidney disease, Hypertension, Blood pressure.

\section{RESUMEN}

Objetivo: Enriquecer la literatura, sintetizar y aclarar la relación de la Enfermedad Renal Crónica (ERC) con la hipertensión. Métodos: Esta revisión utilizó las bases de datos "PubMed" y "Scielo" para buscar los descriptores de "Función renal", "Hipertensión", 'Kidney Función ", "Insuficiencia renal, crónica" y " crónica Kidney Diasease" asociados a operadores booleanos, encontrando 1116 artículos, seleccionaron 20. Resultados: La ERC presenta una elevada mortalidad debido a su carácter silencioso. Caracteriza por anomalías funcionales, estructuras renales durante más de tres meses. La Tasa de Filtración Glomerular se utiliza para el diagnóstico y la estadificación, ya que las principales etiologías, la hipertensión y la diabetes, son foco para el tratamiento de la ERC. Sin una explicación fiable, hay estudios que informan sobre el tratamiento intensivo de la presión y el uso de hidralazina empeoran la función renal. Los antihipertensivos, los fármacos modificadores de los lípidos para el control de la glucemia, clásicos como terapia modificadora de la progresión. Consideraciones finales: Es esencial hacer más hincapié en el cribado y el diagnóstico precoz para mejorar la salud de los pacientes, especialmente de aquellos con comorbilidades. Se necesitan estudios para dilucidar su relación con la hipertensión, el tratamiento intensivo de la presión y otras asociaciones.

Palabras clave: Enfermedad renal crónica, Hipertensión, Presión sanguínea.

\section{INTRODUÇÃO}

A Doença Renal Crônica (DRC) é a perda progressiva da função renal que ocorre ao longo de vários meses e anos, resultando na diminuição da capacidade dos rins de processar resíduos no sangue e realizar outras funções. Inicialmente, a Taxa de Filtração Glomerular (TFG) e albuminúria diminuem ligeiramente, podendo progredir à Doença Renal em Estágio Terminal (ESRD) ou insuficiência renal (GORO KK, et al., 2019).

Historicamente, a DRC foi categorizada por sua causa, que era predominantemente diabetes, hipertensão, glomerulonefrite ou doença renal policística (TAYLOR KS, et al., 2019). O desenvolvimento da DRC está frequentemente associado à hipertensão e diabetes mellitus, que são as principais causas de insuficiência renal crônica em pacientes em programa de diálise (MOURA ECSC, et al., 2017).

A Hipertensão Arterial Sistêmica (HAS) foi identificada como o principal fator de risco para mortalidade em todo o mundo e um dos mais importantes fatores de risco para DRC. As alterações na pressão arterial podem ocorrer sob a influência de vários fatores, como idade, aumento da rigidez vascular, atividade do sistema nervoso simpático e não adesão ao tratamento ( $(\mathrm{I}$ H, et al., 2020). A hipertensão é um importante marcador de risco para o declínio da função renal, uma vez que a Pressão Arterial (PA) elevada está ligada ao início e à progressão da DRC, juntamente com outros fatores de risco, como diabetes, hiperlipidemia, obesidade e tabagismo (JEFFERS BW, et al., 2017).

Apesar da HAS já ser bem definida como uma das causas para a DRC, muitos estudos relatam uma incerteza sobre qual deveria ser a meta ideal de pressão arterial para prevenir o decaimento da função renal. Ainda, existem divergências sobre o tratamento intensivo da pressão, uma vez que há estudos com resultados benéficos e outros que não apresentaram nenhum benefício para o paciente portador de injúria renal crônica (CHEUNG AK, et al., 2017).

A detecção precoce e o tratamento podem prevenir ou minimizar as complicações associadas à DRC; no entanto, a maioria dos casos de DRC não foi clinicamente reconhecida principalmente devido à falta de conhecimento dos pacientes sobre a doença e os fatores de risco associados. O rastreamento de indicadores clínicos de disfunção renal é fundamental para a detecção precoce de pacientes com risco de DRC; além disso, é imprescindível aumentar a conscientização dos pacientes a fim de modificar seu estilo de vida no sentido de prevenir a ocorrência da doença (GORO KK, et al., 2019). 
Há um aumento alarmante da prevalência de DRC globalmente associada à morbidade e mortalidade significativas. A prevalência de DRC na população geral é de $13,4 \%$ globalmente, enquanto a prevalência combinada é de $10,1 \%$ na população geral, $24,7 \%$ em hipertensos e $16,6 \%$ entre pacientes com diabetes mellitus na África. De acordo com a carga global de estudo da doença em 2015, a DRC foi uma das doenças que teve um aumento mais rápido como uma das principais causas de morte, tendo uma diferença positiva de 31,7\% entre 2005 a 2015 (GORO KK, et al., 2019).

A Doença Renal Crônica e o declínio da função renal estão relacionados a altos custos de saúde e resultados terapêuticos insatisfatórios, sendo consideradas importantes preocupações de saúde pública. (JEFFERS BW, et al., 2017). Dado o contexto apresentado e a clara relevância do tema para a comunidade médica, essa revisão teve como objetivo enriquecer a literatura atual a respeito do assunto apresentado, sintetizar as principais informações sobre a DRC e esclarecer a sua relação com a hipertensão.

\section{MÉTODOS}

Para a elaboração do presente trabalho, caracterizado como uma revisão integrativa, buscou-se artigos nas bases de Scientific Electronic Library Online (Scielo) e Pubmed, com os seguintes Descritores em Ciências da Saúde (DECS): "Renal Function", "Hypertension", "Kidney Function", "Renal Insufficiency, Chronic" e "Chronic Kidney Disease". Foram encontrados, ao total, 1116 resultados e selecionados 20 deles para compor esse artigo (Figura 1). Além disso, esses descritores foram associados entre si pelos operadores booleanos.

Como filtros, foram utilizados artigos nos idiomas inglês, português e espanhol e que tivessem sido publicados no período de 1 de Janeiro de 2015 até os dias atuais, sendo a busca feita até o dia 13 de Julho de 2021. Ainda, foram usados os seguintes filtros: ensaio clínico, ensaio controlado randomizado e revisão sistemática. Como critérios de inclusão, selecionaram-se artigos que continham informações sobre a abordagem geral da DRC e a sua relação com a HAS. Excluíram-se artigos que não tinham relevância ou que não tratavam sobre o tema.

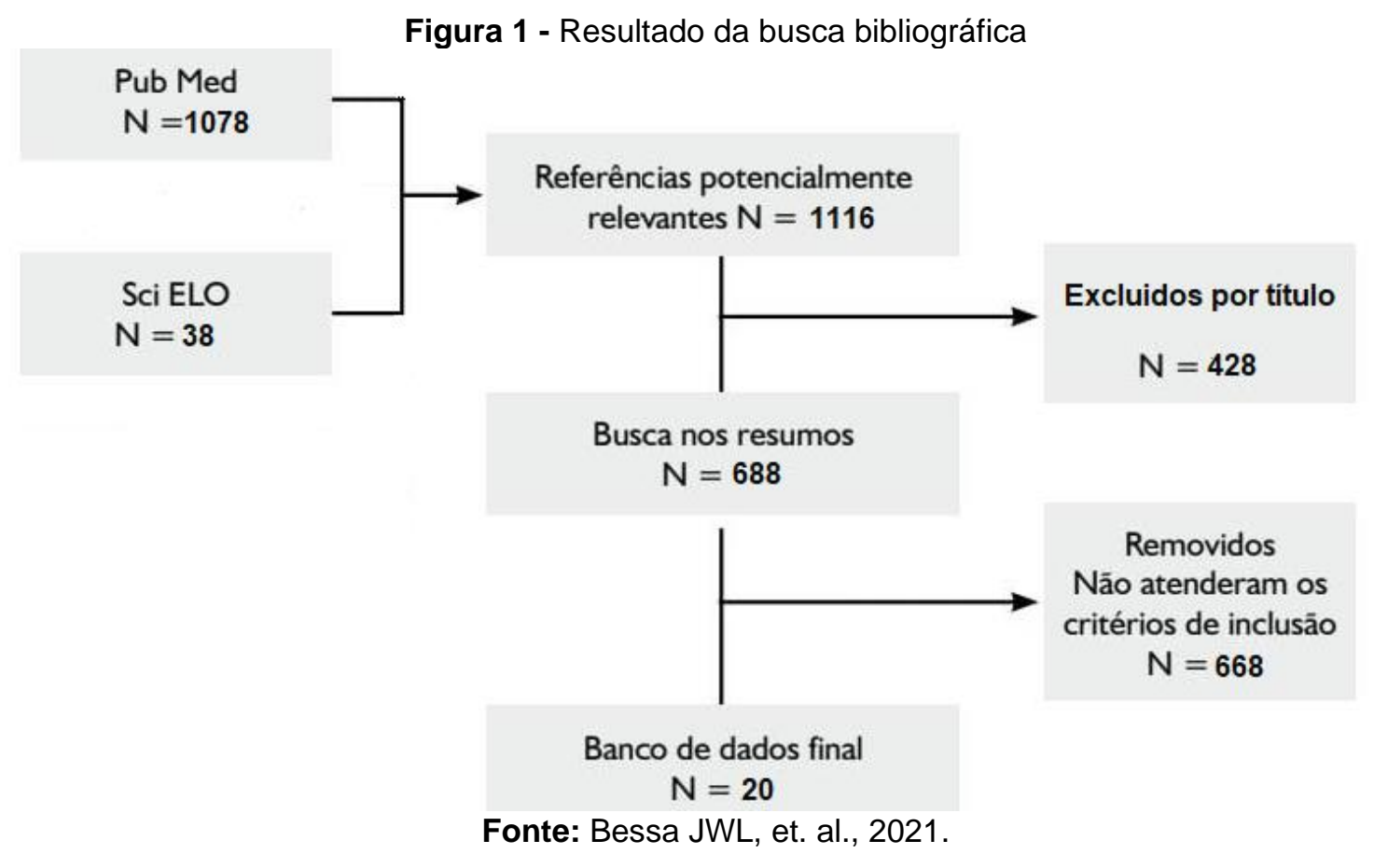

\section{RESULTADOS}

Após a busca nas bases de dados sobre o tema DRC e sua relação com a Hipertensão, foram encontrados 1116 artigos, selecionados os publicados entre 2015 e 2021 e utilizados 20 para compor esta revisão. Os artigos selecionados estão representados no Quadro 1. 
Quadro 1 - Síntese dos principais achados sobre Doença Renal Crônica dentre os 20 artigos selecionados

$\mathbf{N}$ Autores (Ano)

1 AGGARWAL R, et al. (2019) Esse é um estudo do tipo Ensaio Clínico Randomizado que teve como objetivo fazer uma comparação da mortalidade por todas as causas em pacientes com
DRC, direcionando para a pressão arterial sistólica menor que $130 \mathrm{mmHg}$ versus menor que $140 \mathrm{mmHg}$. Em uma análise primária, a taxa de mortalidade por todas as causas teve uma tendência a melhores resultados com o tratamento intensivo da PA.

$2 \quad$ ALMEIDA FAD, et al. (2015)

Trata-se de um estudo caso-controle que teve como objetivo principal avaliar se há agregação familiar da DRC em família de indivíduos em terapia renal substitutiva secundária a HAS ou DM. A conclusão do trabalho foi que as famílias de indivíduo com DRC em terapia renal substitutiva tinham razão de chance de 2,35 (IC95\% 1,42-3,89; $p<0,001)$ de ter DRC versus o grupo controle.

3 CASTRO TLB, et al. (2020)

Trata-se de um estudo transversal com o objetivo de identificar a prevalência da TFGe alterada e fatores associados em pacientes com DM e HAS. Registrou uma falta de acompanhamento do início e desenvolvimento da DRC nas primeiras fases, causando uma abordagem tardia e ações limitadas. Concluiu uma alta prevalência da TFGe sem registro no prontuário que direcionassem para uma abordagem terapêutica da DRC.

Esse trabalho é caracterizado como um estudo randomizado que dividiu 1646 participantes com DRC em dois grupos, cuja metas pressóricas sistólicas deveriam ser menores que $120 \mathrm{mmHg}$ (grupo intensivo) e menor que $140 \mathrm{mmHg}$ (grupo padrão), respectivamente. Esses grupos foram acompanhados por 3,3 anos. Conclui-se que o grupo intensivo teve uma taxa mais baixa de todas as causas de morte, além de maior redução da TFGe. Assim, houve uma redução nas taxas de eventos cardiovasculares e causas de morte.

4 CHEUNG AK, et al. (2017)

Consiste em um estudo longitudinal, do tipo coorte prospectivo e quantitativo, que teve como objetivo analisar a relação entre variações do clearance de

5 DUARTE TTDP, et al. (2020) creatinina $(\mathrm{CICr})$ e os fatores clínicos de pacientes internados em clínica médica. Como resultado, obteve que a maioria tinha HAS e DM, além de que a idade avançada e maior tempo de internação hospitalar se associaram a piora da função renal.

$6 \quad$ GORO KK, et al. (2019)

Esse estudo consiste em um ensaio clínico com o objetivo de avaliar o conhecimento, a prevalência e os fatores de risco para a DRC em pacientes com HAS e DM. A partir disso, concluiu que a maioria dos pacientes estudados tinham baixo nível de conhecimento e alta prevalência de injúria renal crônica.

Trata-se de um estudo controlado e randomizado que teve como objetivo avaliar o impacto da função renal, usando a TFGe, na variabilidade da pressão arterial. $\mathrm{Na}$ análise dos resultados, observou-se que os pacientes com menor TFGe apresentavam maior PAS. Conclui-se então que pacientes com declínio de função renal têm uma piora na pressão arterial sistólica, mesmo após ajustes para fatores de risco.

7 JEFFERS BW, et al. (2017)

Consiste em um estudo randomizado e controlado que teve como objetivo avaliar um novo método (urinálise em smartphone) para o rastreamento de proteinúria em pacientes com HAS. Teve como resultado um aumento da adesão ao rastreio para proteinúria e uma possibilidade de diagnóstico precoce para a DRC.

LEDDY J, et al. (2019)

Trata-se de uma revisão sistemática e metanálise que tinha como objetivo avaliar a associação entre a variabilidade da pressão arterial de visita a visita e o risco de ter DRC. Como resultados, obteve que os pacientes tinham maior risco de ter a patologia com altas variabilidades da pressão arterial sistólica. Então, concluíram que o aumento dessa variabilidade era um fator de risco independente para DRC.

Consiste em um estudo clínico randomizado de pacientes com DRC que atingiram o valor da pressão arterial sistólica alvo para seus respectivos grupos (intensivo menor que $120 \mathrm{mmHg}$ e padrão menor que $140 \mathrm{mmHg}$ ). Como resultado, obteve que o grupo intensivo teve risco maior para eventos adversos hipoperfusos e que a variabilidade da pressão arterial diastólica (PAD) previu esses eventos. Assim, concluiu-se que a variação da PAD prediz independentemente os resultados cardiovasculares e eventos adversos em pacientes com DRC.

REAMed | Vol.1(1) | DOI: https://doi.org/10.25248/REAMed.e8904.2021

Página 4 de 9 


\begin{tabular}{|c|c|c|}
\hline $\mathbf{N}$ & Autores (Ano) & Principais achados \\
\hline 11 & $\begin{array}{l}\text { MOURA ECSCD, et al. } \\
(2017)\end{array}$ & $\begin{array}{l}\text { Trata-se de um estudo transversal com cardiologistas e endocrinologistas respondendo questionários sobre a prevenção da DRC em pacientes hipertensos } \\
\text { e diabéticos. O objetivo deste estudo foi verificar o conhecimento, as indicações e as práticas na prevenção dessa patologia. Como resultado, obteve uma } \\
\text { baixa solicitação de exames de rotina para o acompanhamento da função renal e a presença de comprometimento renal em alguns dos seus pacientes. }\end{array}$ \\
\hline 12 & OBI Y, et al. (2018) & $\begin{array}{l}\text { Consiste em um estudo de coorte prospectivo em adultos hipertensos e sem diabetes com TFGe }>20 \mathrm{ml} / \mathrm{min} / 1,73 \mathrm{~m}^{2} \text {. Os pacientes foram divididos em grupos } \\
\text { de controle intensivo da PA (menor que } 120 \mathrm{mmHg} \text { ) ou controle padrão (menor que } 140 \mathrm{mmHg} \text { ). Como resultado, observou-se que dependendo do valor da } \\
\text { TFGe, o controle da PA pode ser benéfico ou não em eventos adversos. Como conclusão geral, afirma que o controle intensivo da PA mostrou pouco ou } \\
\text { nenhum benefício, podendo até ser prejudicial para pacientes com DRC moderada a avançada. }\end{array}$ \\
\hline 13 & OKIN PM, et al. (2016) & $\begin{array}{l}\text { Esse estudo clínico randomizado teve como objetivo avaliar a TFG em relação à pressão arterial sistólica (PAS) média durante o tratamento de pacientes } \\
\text { hipertensos. Como resultado, observou-se que as pessoas com PAS média menor que } 130 \mathrm{mmHg} \text { tiveram a TFG basal menor do que aquelas com PAS } \\
\text { maior que } 130 \mathrm{mmHg} \text {. No estudo ainda observou que, nesses pacientes onde a PAS era mais baixa, houve uma redução mais lenta da TFG no decorrer do } \\
\text { tempo. }\end{array}$ \\
\hline 14 & $\begin{array}{l}\text { PASSOS VMDA, et al. } \\
(2018)\end{array}$ & $\begin{array}{l}\text { Trata-se de uma coorte multicêntrica com o objetivo de investigar a associação entre DRC e desempenho cognitivo em brasileiros de } 35-74 \text { anos. Como } \\
\text { resultado, obteve que o desempenho cognitivo reduzido estava presente em } 15,8 \% \text { dos pacientes estudados e neles havia ainda valores de TFG } \\
\text { discretamente diminuídos em relação aqueles com desempenho cognitivo normal. Como conclusão, afirma que o desempenho cognitivo permanece } \\
\text { preservado até a função renal atingir piora significativa. }\end{array}$ \\
\hline 15 & $\begin{array}{l}\text { RADHAKRISHNA V, et al. } \\
\qquad(2018)\end{array}$ & $\begin{array}{l}\text { Consiste em um estudo transversal com o objetivo de avaliar a etiologia, a apresentação, presença de malformações congênitas renais ou urinárias e dano } \\
\text { renal em crianças com rim solitário funcional. Como conclusão, teve que essas crianças apresentavam alta morbidade e a necessidade de acompanhamento } \\
\text { periódico. }\end{array}$ \\
\hline 16 & $\begin{array}{l}\text { RIGONATTO MCL e } \\
\text { MAGRO MCDS (2018) }\end{array}$ & $\begin{array}{l}\text { Trata-se de um estudo observacional, longitudinal e prospectivo com o objetivo de identificar pacientes hipertensos e diabéticos com risco de desenvolver } \\
\text { lesão renal aguda. De resultados, obteve que um total de } 23,2 \% \text { dos usuários evoluíram para comprometimento renal, além de idade e índice de massa } \\
\text { corporal influenciar na piora da função renal. Assim, concluiu-se que um quarto dos pacientes evoluiu para comprometimento da função renal. }\end{array}$ \\
\hline 17 & $\begin{array}{l}\text { SCHAEFER JCF, et al. } \\
(2015)\end{array}$ & $\begin{array}{l}\text { Esse estudo transversal de base populacional teve como objetivo estimar a função renal e identificar fatores associados com a TFG. Como resultado, notou } \\
\text { uma diferença significativa da TFG relacionada a idade e obesidade, sendo que quanto maior seriam a idade e obesidade, menor seria a TFG. Em conclusão, } \\
\text { teve que a maior parte dos indivíduos possuía essa taxa em valores normais e apenas } 1,4 \% \text { tinham disfunção de grau moderado ou superior. }\end{array}$ \\
\hline 18 & TAYLOR KS, et al. (2019) & $\begin{array}{l}\text { Consiste em uma revisão sistemática e metanálise que tem como objetivo avaliar os efeitos de intervenções medicamentosas que podem modificar a } \\
\text { progressão da DRC em adultos nos estágios } 3 \text { e } 4 \text {. Como resultado, obteve que a TFGe foi } 6 \% \text { maior em pacientes que fazem controle glicêmico e } 4 \% \text { maior } \\
\text { naqueles que fazem uso de drogas modificadores de lipídio. Não houve diferença com drogas anti-hipertensivas. Como conclusão, afirma que as drogas para } \\
\text { controle glicêmico e de lipídios podem retardar a progressão da DRC. }\end{array}$ \\
\hline 19 & VAKILZADEH N, et al. (2015) & $\begin{array}{l}\text { Trata-se de um estudo prospectivo randomizado que teve como objetivo comparar um inibidor direto da renina (aliscireno) ao diurético hidroclorotiazida na } \\
\text { capacidade de modular a oxigenação do tecido renal em pacientes hipertensos. Como resultado, não houve alterações significativas no final do estudo. No } \\
\text { entanto, em análise dos grupos, teve que o aliscireno aumenta ligeiramente a oxigenação do córtex renal e diminuiu na região medular, enquanto o } \\
\text { hidroclorotiazida teve uma diminuição geral }\end{array}$ \\
\hline 20 & $\begin{array}{l}\text { ZUCKERMAN R, et al. } \\
\qquad(2018)\end{array}$ & $\begin{array}{l}\text { Consistem em dois relatos de casos que apresentaram lesão renal aguda (LRA), proteinúria e hematúria após o uso de hidralazina (vasodilatador) para } \\
\text { tratamento da hipertensão. Na biópsia renal apresentou glomerulonefrite rapidamente progressiva e a hidralazina foi interrompida. Um dos pacientes precisou } \\
\text { de diálise e o outro precisou de hemodiálise devido a LRA grave }\end{array}$ \\
\hline
\end{tabular}

Fonte: Bessa JWL, et. al., 2021. 


\section{DISCUSSÃO}

A DRC é considerada como um grande problema mundial de saúde pública. As doenças que afetam o rim e o trato urinário causam, aproximadamente, 850 milhões de mortes anuais no planeta e a sua incidência aumenta cada vez mais, por volta de $8 \%$ ao ano (CASTRO TLB, et al., 2020). A DRC é uma alteração de saúde silenciosa na maior parte dos casos, mesmo com o diagnóstico sendo basicamente feito com a história clínica e exames laboratoriais, ou seja, sem demanda alta de custos para isso. Contudo, na ausência de detecção precoce, a doença cursa progressivamente e pode acarretar, algumas vezes, em perda total da função do órgão (SCHAEFFER JCF, et al., 2014).

De acordo com as últimas diretrizes que avalia o manuseio e prática clínica, entende-se por DRC as anormalidades funcionais e/ou estruturais dos rins, durando por mais de três meses e com consequências para a saúde do paciente. A TFG e a Taxa de Filtração Glomerular estimada (TFGe), que é uma adaptação da TFG, são utilizadas para diagnóstico e classificação da DRC. Para calcular a TFG, deve-se levar em consideração a interferência de alguns fatores, tais como idade, sexo e raça. Além disso, ela pode alterar por outras causas, a exemplo as Doenças Crônicas Não Transmissíveis (DCNT) como a HAS (DUARTE TTDP, et al., 2020). Ainda, essa medida pode ser obtida e identificada em equações, utilizando os valores de creatinina sérica (CASTRO TLB, et al., 2020).

As classificações de comprometimento renal foram estabelecidas após o cálculo do clearance de creatinina estimado através da equação feita pela Colaboração de Epidemiologia de Doença Renal Crônica (CKD-EPI). Essa equação utiliza como marcador principal a creatinina sérica para fazer esse cálculo. Sendo assim, a depender da gravidade de agressão renal, o paciente pode ser classificado em estágio G1(função renal normal), estágio G2 (função renal discretamente reduzida), estágio G3a (comprometimento renal leve a moderadamente diminuído), estágio G3b (função renal moderadamente a severamente diminuídas), estágio G4 (severamente diminuído) e grupo G5 (falência renal) (DUARTE TTDP, et al., 2020).

Embora os critérios para diagnóstico de DRC sejam bem estabelecidos pela literatura, devido ao fato da doença ter progressão assintomática ou oligossintomática, há uma dificuldade de fazer o diagnóstico precoce e evitar mais danos para o paciente. Assim, é comum que o diagnóstico seja feito apenas no estágio avançado da doença, quando há limitações de ações visando a preservação das funções renais e combatendo o aparecimento de sintomas. No início da doença, quando aparece algum sinal e/ou sintomas, eles são inespecíficos, tais como náuseas, vômitos, inapetência, edema e aumento da PA. Por ser inespecífico, não direcionam de forma direta para o diagnóstico de doença renal (CASTRO TLB, et al., 2020). Já na fase mais avançada, há estudos que relatam até declínio do desempenho cognitivo correlacionado com a diminuição da TFG (PASSOS VMDA, et al., 2018).

As principais causas da DRC são a HAS, Diabetes Mellitus (DM) e as glomerulopatias. Além disso, pode citar como outras possíveis etiologias as malformações congênitas renais e/ou urinárias, bem como síndrome metabólica e neoplasias. A HAS foi identificada como o principal fator de risco para mortalidade em todo o mundo e um dos mais importantes fatores de risco para DRC. As flutuações da PA podem ocorrer sob a influência de vários fatores, como idade, aumento da rigidez vascular, atividade do sistema nervoso simpático e não adesão ao tratamento. Sabendo então da influência da hipertensão na DRC, recomenda-se, para os pacientes que apresentam HAS, um rastreio de proteinúria para aumentar as estratégias de identificação precoce da injúria renal crônica (LEDDY J, et al., 2019; LI H, et al., 2020; RADHAKRISHNA V, et al., 2018).

Há fortes evidências que apoiam uma associação bidirecional entre PA e função renal deprimida. Em ambos os estudos populacionais de indivíduos sem evidência basal de DRC e pacientes com DRC, níveis crescentes de Pressão Arterial Sistólica (PAS) no início do estudo foram associados ao aumento do risco de ESRD (RIGONATTO MCL e MAGRO MCS, 2017). Apesar das causas de DRC terminal serem múltiplas, a hipertensão arterial e o diabetes mellitus tipo 2 são, há muito tempo, as etiologias mais prevalentes e correspondem a $63,5 \%$ dos casos no Brasil e em torno de $70 \%$ dos casos nos Estados Unidos (AIMEIDA FA, et al., 2015).

A literatura tem fortes evidências demonstrando que pacientes com diabetes mellitus e hipertensão sofrem alterações funcionais e estruturais em órgãos-alvo como coração, cérebro, rins e vasos sanguíneos. A 
predisposição e os riscos de disfunção renal devem ser identificados e minimizados. Indivíduos com essas comorbidades depois de seis meses, acumulam risco para desenvolver Lesão Renal Aguda (LRA), pois nesse período ocorrem perdas no mecanismo de autorregulação do fluxo sanguíneo renal, o que pode levar à redução da perfusão renal (RIGONATTO MCL e MAGRO MCS, 2017). Além disso, vale ressaltar que o efeito deletério da HAS no tecido renal apresenta como alguns mecanismos patogênicos a disfunção endotelial, a inflamação, o aumento da pressão intraglomerular, estresse oxidativo e proteinúria (VAKILZADEH N, et al., 2015).

Quatro classes diferentes de medicamentos podem modificar a progressão da DRC: antihipertensivos, medicamentos modificadores de lipídios, medicamentos de controle glicêmico em pacientes com diabetes e bicarbonato de sódio. O bicarbonato de sódio aborda a acidose metabólica, uma complicação prevalente na DRC em estágio moderado e tardio, e pode oferecer um tratamento simples e de baixo custo para retardar a progressão da DRC. Os efeitos dos demais medicamentos na DRC são secundários, visto que esses medicamentos são usados principalmente para tratar as causas primárias, como a hipertensão, a hipercolesterolemia e/ou o DM (TAYLOR KS, et al., 2019).

Outra opção seria as Terapias de Substituição Renal (TSR) no estágio final da doença (taxa de filtração glomerular $<15 \mathrm{ml} / \mathrm{min} / 1,73 \mathrm{~m} 2$ ). Elas incluem a hemodiálise, a diálise peritoneal e o transplante de rim. A prevalência de pacientes em diálise no Brasil aumentou de 333 Pacientes Por Milhão de Habitantes (PMH) em 2004 para $483 \mathrm{PMH}$ em 2010 e espera-se que continue a aumentar a uma taxa anual de $6,5 \%$. Mais de $85 \%$ dos pacientes com TSR no Brasil são tratados pelo Sistema Único de Saúde (SUS), que atualmente está à beira da saturação e com dificuldades em atender a demanda de pacientes com DRC em estágio terminal (ALMEIDA FAD, et al., 2015).

Ao tratar pacientes hipertensos, níveis mais baixos de PAS alcançados devem estar associados a menos piora da função renal ao longo do tempo (OKIN P.M, et al, 2016). No entanto, é imprescindível ressaltar que há estudos relatando efeitos adversos do uso de hidralazina no tratamento da hipertensão como um potente causador de lesão renal aguda e DRC (ZUCKERMAN R, et al., 2018). É consenso que o diagnóstico rápido e efetivo, além de ações preventivas na tentativa de buscar ativamente e conscientizar os pacientes em risco de desenvolvimento de DRC ou aqueles já com a patologia, podem preservar ou delongar a evolução da doença para fases terminais (CASTRO TLB, et al., 2020).

No entanto, as descobertas foram confusas quanto aos efeitos de um controle mais intensivo da PA sobre a taxa de progressão da doença renal. Entre os pacientes com DRC, a randomização para redução mais intensa da PAS não diminuiu significativamente a progressão da doença renal no estudo de Modificação da Dieta na Doença Renal (MDRD) ou no Estudo Africano Americano de Doença Renal e Hipertensão (AASK) (OKIN PM, et al., 2016).

Ensaios clínicos randomizados anteriores comparando a redução da PA intensiva e padrão em pacientes com DRC sem diabetes não mostraram nenhum benefício geral do tratamento intensivo da PA em seus resultados renais primários. Estudos observacionais e ensaios clínicos de pequeno ou moderado tamanho que incluíram pacientes com leve a moderada DRC produziram resultados mistos, com alguns sugerindo uma relação linear direta entre a PA e essa patologia, enquanto outros sugerem uma relação em "forma de J" (Grande queda inicial seguida por forte elevação), dependendo do parâmetro específico da PA e do tipo de DRC estudado (CHENG AK, et al., 2017).

Digno de nota, várias meta-análises recentes apoiam a conclusão de que uma redução mais intensa da PA parece reduzir o risco de Doença Renal Terminal (DRT) entre os pacientes com DRC com proteinúria, mas não naqueles sem proteinúria. As análises pós-estudo com acompanhamento de longo prazo de controle mais intensivo da PA em pacientes com DRC demonstraram uma redução estatisticamente significativa de $32 \%$ no risco de DRT em MDRD (OKIN PM, et al., 2016).

O Systolic Blood Pressure Intervention Trial (SPRINT) foi projetado para comparar os efeitos da redução intensiva da PA (PA sistólica [PAS] $<120 \mathrm{mmHg}$ ) e do controle da PA padrão (PAS $<140 \mathrm{mmHg}$ ) nos desfechos clínicos. É o maior ensaio randomizado até o momento para avaliar o efeito de diferentes alvos de PA sobre doenças cardiovasculares (DCV) e resultados renais em pacientes com DRC. No geral, a redução 
intensiva da PAS resultou em taxas mais baixas do desfecho composto primário de DCV e morte por todas as causas; o efeito do tratamento não foi modificado pela presença ou ausência de DRC no início do estudo. Além disso, não houve diferença na incidência do resultado renal composto principal definido como $\geq 50 \%$ de redução na eTFG da linha de base ou ESRD (AGGARWAL R, et al., 2019; CHENG AK, et al., 2017).

Em suma, a análise da variabilidade da pressão arterial sistólica, medidas em visitas diferentes, está correlacionada com um aumento da DRC em pacientes com diferenças maiores entre esses valores. A explicação para isso é que a rigidez arterial, a inflamação crônica e a alta atividade simpática comumente observada em pacientes com injúria renal crônica são mecanismos potenciais para essas flutuações. Em um dos resultados obtidos, conclui-se que, para cada aumento de $1 \mathrm{mmHg}$ na pressão arterial sistólica, o Risco Relativo (RR) de ter DRC foi de 1.05 (IC 95\%: 1.03-1.07). Apesar de já conhecer a influência dessa variabilidade da pressão arterial, teve estudos que demonstraram uma incidência maior de eventos adversos graves com o tratamento intensivo da pressão arterial para valores menores que $120 \mathrm{mmHg}$ (MEZUE K, et al., 2018; LI H, et al., 2020; OBI Y, et al., 2018).

\section{CONSIDERAÇÕES FINAIS}

A DRC, como uma patologia silenciosa na maior parte do tempo, apresenta diversas etiologias, sendo a HAS intrinsecamente associada e um dos principais fatores de risco para a DRC, embora necessite de mais estudos para explicar com fidedignidade a causa para essa questão. Com isso, devido à possibilidade direta de afetar a qualidade de vida do paciente e avanço rápido, necessita de medidas e ações para rastreio e diagnóstico precoce para não trazer prejuízos para a vida de seu portador, incluindo a morte. Além disso, devido a algumas questões não esclarecidas com evidente embasamento científico, como o controle intensivo da PA em pacientes com a injúria renal crônica, é necessário que mais estudos sejam feitos para elucidar essas relações. Assim, haverá um atendimento eficaz para saúde da população, contribuindo para a qualidade de vida dos pacientes acometidos por esse quadro clínico.

\section{REFERÊNCIAS}

1. AGGARWAL R, et al. Mortality Outcomes With Intensive Blood Pressure Targets in Chronic Kidney Disease Patients. Hypertension, 2019; 73(6): 1275-1282.

2. ALMEIDA FAD, et al. Family clustering of secondary chronic kidney disease with hypertension or diabetes mellitus. A case-control study. Ciência \& Saúde Coletiva, 2015; 20(2).

3. CASTRO TLB, et al. Função renal alterada: prevalência e fatores associados em pacientes de risco. Revista Cuidarte, $2020 ; 11(2)$.

4. CHEUNG AK, et al. Effects of Intensive BP Control in CKD. Journal of the American Society of Nephrology: JASN, 2017; 28(9): 2812-2823.

5. DUARTE TTDP, et al. Influência de fatores clínicos na lesão renal aguda. Ciencia y enfermería, 2020; 26(1).

6. GORO KK, et al. Patient Awareness, Prevalence, and Risk Factors of Chronic Kidney Disease among Diabetes Mellitus and Hypertensive Patients at Jimma University Medical Center, Ethiopia. BioMed Research International, 2019; 1(1).

7. JEFFERS BW, ZHOU D. Relationship Between Visit-to-Visit Blood Pressure Variability (BPV) and Kidney Function in Patients with Hypertension. Kidney and Blood Pressure Research, 2017; 42(4): 697-707.

8. LEDDY J, et al. Improving proteinuria screening with mailed smartphone urinalysis testing in previously unscreened patients with hypertension: a randomized controlled trial. BMC Nephrology, 2019; 20(1): 132.

9. LI H, et al. Visit-to-visit blood pressure variability and risk of chronic kidney disease: A systematic review and metaanalyses. PLoS One, 2020; 15(5).

10. MEZUE K, et al. Blood Pressure Variability Predicts Adverse Events and Cardiovascular Outcomes in Chronic Kidney Disease: A Post-Hoc Analysis of the SPRINT Trial. American Journal of Hypertension, 2018; 31(1): 48-52.

11. MOURA ECSCD, et al. Knowledge regarding the prevention of chronic kidney disease in hypertensive and diabetic patients: a cross-sectional study. Fisioterapia em Movimento, 2017; 30(1).

12. $\mathrm{OBI} \mathrm{Y}$, et al. Estimated glomerular filtration rate and the risk-benefit profile of intensive blood pressure control amongst nondiabetic patients: a post hoc analysis of a randomized clinical trial. Journal of Internal Medicine, 2018; 283(3): 314327. 
13. OKIN PM, et al. Impact of achieved systolic blood pressure on renal function in hypertensive patients. European Heart Journal - Quality of Care and Clinical Outcomes, 2016; 2(4): 271-276.

14. PASSOS VMDA, et al. First stages chronic kidney disease have mild effects on cognitive performance. Results of a 15,105 brazilian adult baseline cohort. Brazilian Journal of Nephrology, 2018; 40(1).

15. RADHAKRISHNA V, et al. Solitary functioning kidney in children: clinical implications. Brazilian Journal of Nephrology, 2018; 40(3).

16. RIGONATTO MCL, MAGRO MCDS. Risco para lesão renal aguda na atenção primária à saúde. Revista Brasileira de Enfermagem, 2018; 71(1).

17. SCHAEFER JCF, et al. Estimativa da função renal na população de 18 a 59 anos da cidade de Tubarão-SC: Um estudo de base populacional. Brazilian Journal of Nephrology, 2015; 37(2).

18. TAYLOR KS, et al. Effects of antihypertensives, lipid-modifying drugs, glycaemic control drugs and sodium bicarbonate on the progression of stages 3 and 4 chronic kidney disease in adults: a systematic review and metaanalysis. BMJ Open, 2019; 9(9): 1-15.

19. VAKILZADEH N, et al. Comparative Effect of a Renin Inhibitor and a Thiazide Diuretic on Renal Tissue Oxygenation in Hypertensive Patients. Kidney and Blood Pressure Research, 2015; 40(5): 542-554.

20. ZUCKERMAN R, et al. Hydralazine-associated adverse events: a report of two cases of hydralazine-induced ANCA vasculitis. Brazilian Journal of Nephrology, 2018; 40(2). 